\title{
Neurofilament Proteins in Y-Cells of the Cat Lateral Geniculate Nucleus: Normal Expression and Alteration with Visual Deprivation
}

\author{
Martha E. Bickford, ${ }^{1}$ William Guido, ${ }^{2}$ and Dwayne W. Godwin ${ }^{3}$ \\ ${ }^{1}$ Department of Anatomical Sciences and Neurobiology, University of Louisville, School of Medicine, Louisville, Kentucky \\ 40292, 'Department of Cell Biology and Anatomy, Louisiana State University Medical Center, New Orleans, Louisiana \\ 70112, and ${ }^{3}$ Department of Neurobiology and Anatomy, Wake Forest University School of Medicine, Winston-Salem, \\ North Carolina 27157-1010
}

\begin{abstract}
We examined neurofilament staining in the normal and visually deprived lateral geniculate nucleus (LGN), using the SMI-32 antibody. This antibody preferentially stains LGN cells that display the morphological characteristics of Y-cells. The soma sizes of SMI-32-stained cells were consistent with those of the overall population of Y-cells, and the Golgi-like staining of their dendrites revealed a radial distribution that often crossed laminar boundaries. Labeled cells were distributed within the $A$ laminae (primarily near laminar borders), the magnocellular portion of the $\mathrm{C}$ laminae, and the medial intralaminar nucleus, but they were absent in the parvocellular $\mathrm{C}$ laminae. Electron microscopic examination of SMI-32-stained tissue revealed that staining was confined to somata, dendrites, and large myelinated axons. Retinal synapses on SMI-32-labeled dendrites
\end{abstract}

were primarily simple axodendritic contacts; few triadic arrangements were observed. In the LGN of cats reared with monocular lid suture, SMI-32 staining was decreased significantly in the A laminae that received input from the deprived eye. Dephosphorylation of the tissue did not alter the cellular SMI-32 staining patterns. Analysis of staining patterns in the $\mathrm{C}$ laminae and monocular zone of the A laminae suggests that changes in the cytoskeleton after lid suture reflect cell class and not binocular competition. Taken together, the results from normal and lid-sutured animals suggest that the cat LGN offers a unique model system in which the cytoskeleton of one class of cells can be manipulated by altering neuronal activity.

Key words: SMI-32; electron microscopy; monocular deprivation; immunocytochemistry; thalamus; cytoskeleton
The SMI-32 antibody stains the nonphosphorylated form of the high-molecular-weight neurofilament protein. It has been used extensively to document cytoskeletal changes in a number of neurological disorders (Sternberger et al., 1985; Troncoso et al., 1986; Hof and Morrison, 1990; Hof et al., 1990; Vickers et al., 1992; Duong and Gallagher, 1994; Gai et al., 1994; Smith et al., 1995; Su et al., 1996). Decreases in neurofilaments, or abnormal phosphorylation of neurofilaments, appear to be frequent consequences of neuronal disease or damage, but the sequence of events leading to these changes is unknown. An understanding of the normal functions of the cytoskeleton, and the potential transformations that occur during neuronal disease, requires an animal model. The lateral geniculate nucleus (LGN) of the cat is an excellent candidate for such a model. Its anatomy and physiology are well characterized, both in the normal condition as well as after experimental manipulations that produce subtle, yet reproducible, changes in neuronal morphology.

The pathway from retina through the LGN is composed of at least two (X and Y), perhaps three (W), morphologically and physiologically distinct neuronal streams. Each is designed to analyze different aspects of the visual scene (Sherman, 1985).

\footnotetext{
Received Feb. 3, 1998; revised May 6, 1998; accepted June 2, 1998.

This work was supported by National Institute of Neurological Diseases and Stroke Grant R29NS35377 and National Science Foundation Grant 97628089 to M.B., National Science Foundation Grant 9396270 to W.G., and National Eye Institute Grant EY11695 to D.G. We thank Martin Boyce for his excellent technical assistance.

Correspondence should be addressed to Dr. Martha E. Bickford, Department of Anatomical Sciences and Neurobiology, 500 South Preston Street, University of Louisville, School of Medicine, Louisville, KY 40292.

Copyright (C) 1998 Society for Neuroscience $0270-6474 / 98 / 186549-09 \$ 05.00 / 0$
}

These pathways develop at different rates and also respond differently to abnormal visual input (Friedlander et al., 1982; Sherman and Spear, 1982; Sur et al., 1982, 1984; Garraghty et al., 1986, 1988). For example, Y-cells seem particularly susceptible to monocular lid suture (MS). After early periods of MS, Y-cells in the LGN develop abnormal receptive field properties and show a reduced soma size and anomalous dendritic morphology. The abnormally thin and tangled dendrites of Y-cells in deprived laminae suggest an alteration in cytoskeletal support. Thus, MS may be a useful model to study how modifications in neuronal activity can alter the organization of neurofilaments.

The aim of the present study was to examine the staining pattern of the SMI-32 antibody in the LGN of normal cats and cats raised with MS. This antibody recently has been shown to stain preferentially the magnocellular layers of the monkey LGN (Gutierrez et al., 1995; Chaudhuri et al., 1996). However, SMI-32 staining has yet to be explored in the cat LGN, where the Y-cell pathway is well characterized and can be manipulated readily by altering visual input.

\section{MATERIALS AND METHODS}

A total of five cats were used for these experiments. Two were normal adult cats. Three other adult cats were reared with monocular lid suture that was performed before eye opening and was maintained until death (for details on lid suture, see Friedlander et al., 1982). The cats were given an overdose of sodium pentobarbital and perfused through the heart with saline, followed by a fixative solution of $4 \%$ paraformaldehyde (one normal and two MS) or 4\% paraformaldehyde and $0.5 \%$ glutaraldehyde (one normal) or $2 \%$ paraformaldehyde and $0.1 \%$ glutaraldehyde (one MS) in $0.1 \mathrm{M}$ sodium phosphate buffer (PB), pH 7.4. Previous studies that used the SMI-32 antibody excluded the use of glutaraldehyde fixation (Campbell and Morrison, 1989). However, we found that, al- 

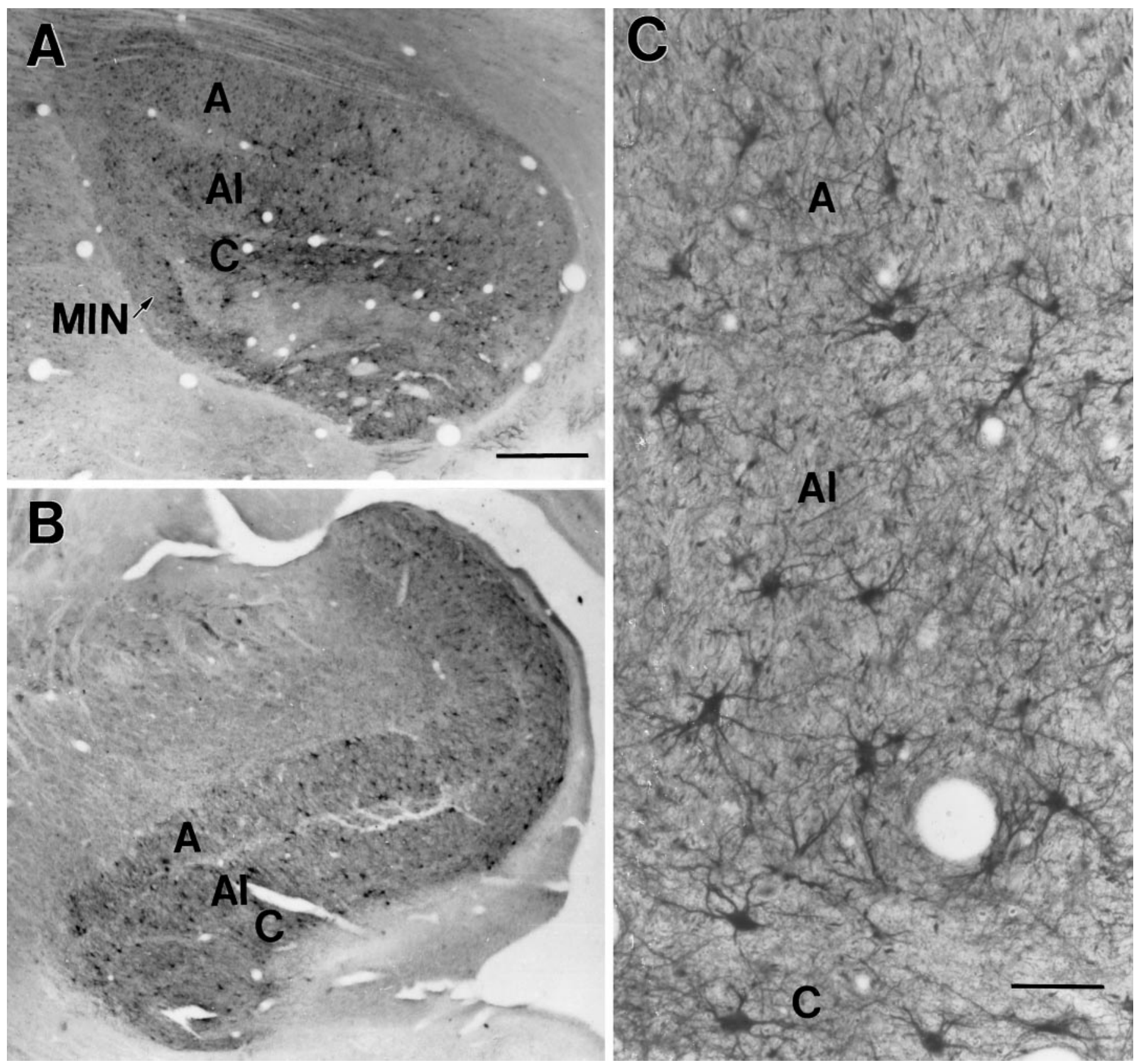

Figure 1. SMI-32-stained cells in the normal LGN match the distribution of Y-cells. Stained cells are located in laminae $A$ and $A 1$, the magnocellular portion of the $\mathrm{C}$ laminae $(C)$, and medial intralaminar nucleus $(M I N)$, as shown in coronal $(A)$ and sagittal $(B)$ sections through the LGN. Higher magnification $(C)$ shows that stained cells are located near laminar borders, and their radially distributed dendritic arbors cross laminar borders. Scale bars: in $A$ (also applies to $B$ ), $1 \mathrm{~mm}$; in $C, 100 \mu \mathrm{m}$.

though the low percentage of glutaraldehyde slightly decreased the staining intensity, the overall staining pattern was similar with each fixation protocol.

After fixation, the brains were removed and cut in the coronal or sagittal plane into $50-\mu \mathrm{m}$-thick sections with a vibratome. Series of sections from all five cats were stained with the SMI-32 antibody. Series of sections from two cats (one normal and one MS) were mounted on slides and stained for Nissl substance. Additional sections from two cats (one normal and one MS) were incubated in alkaline phosphatase (400 $\mu \mathrm{g} / \mathrm{ml}$; Sigma type V II-L, Sigma, St. Louis, MO) in $0.1 \mathrm{M}$ Tris buffer, $\mathrm{pH}$ 8 , for $2 \mathrm{hr}$ at $37^{\circ} \mathrm{C}$, rinsed in Tris buffer, and stained with the SMI-32 antibody as described below.

For immunocytochemistry, sections through the LGN were incubated in $10 \%$ normal goat serum (NGS) in PBS $(0.01 \mathrm{~m} ; 0.9 \% \mathrm{NaCl})$ for $30 \mathrm{~min}$. Then the sections were transferred to a solution of the SMI-32 antibody (monoclonal, made in mouse, Sternberger Monoclonal, Jarrettsville, MD) diluted $1: 5000,1: 10,000$, or $1: 20,000$ in $1 \%$ NGS in PBS with $0.5 \%$ Triton X-100 and incubated overnight with agitation at $4^{\circ} \mathrm{C}$. The next day the sections were rinsed three times (10 min each) in PB and incubated for $1 \mathrm{hr}$ in a 1:100 dilution of biotinylated goat anti-mouse antibody (Vector Laboratories, Burlingame, CA) in 1\% NGS in PBS. Then the sections were rinsed three times (10 min each) in PB and incubated for $1 \mathrm{hr}$ in a 1:100 dilution of a complex of avidin and biotinylated horseradish peroxidase (ABC; Vector Laboratories) in 1\% NGS in PBS. The sections were rinsed three times $(10 \mathrm{~min}$ each) and reacted with nickelenhanced diaminobenzidine solution (Adams, 1981) for $5 \mathrm{~min}$. The sections were rinsed and mounted on slides or prepared for electron microscopy as described below.

For electron microscopy the sections were post-fixed in osmium (2\% in $\mathrm{PB})$ for $1 \mathrm{hr}$, rinsed, dehydrated in an ethanol series, and embedded in 

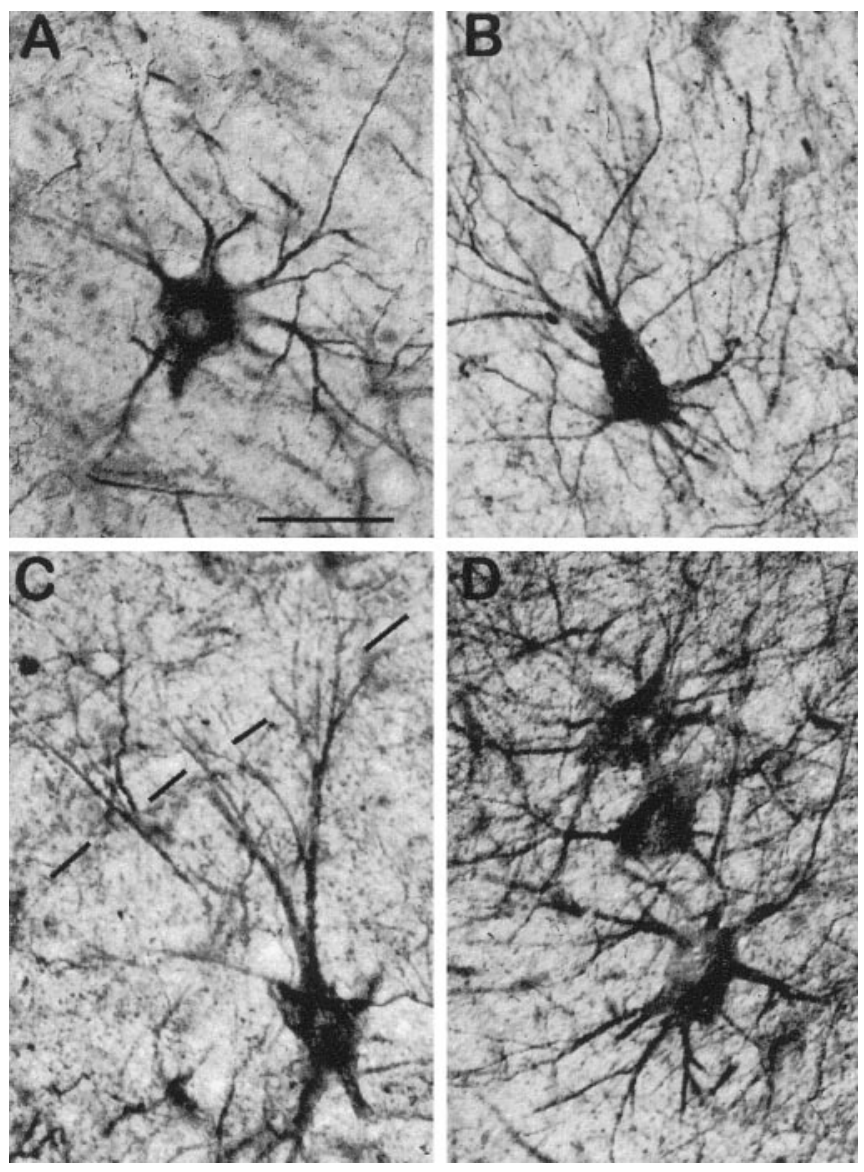

Figure 2. SMI-32-stained cells in the normal LGN display class I morphology. $A$, Cell in lamina A. $B$, Cell in lamina A1. $C$, Cell in lamina A1 extending dendrites into the interlaminar zone (border indicated by the dashed line). $D$, Cells in the medial intralaminar nucleus. Scale bar in $A$ (also applies to $B-D$ ), $50 \mu \mathrm{m}$.

Durcupan resin (Ted Pella, Redding, CA). Sections were cut from the resin blocks on an ultramicrotome, collected on Formvar-coated slot grids, stained with uranyl acetate (10\% in methanol) for $30 \mathrm{~min}$, and examined with a transmission electron microscope. SMI-32-labeled dendrites postsynaptic to retinal terminals were photographed.

Drawings of soma sizes from Nissl- and SMI-32-stained tissue were made with a camera lucida. The areas of these drawings subsequently were measured by a digitizing tablet and SigmaScan computer software (Jandel Scientific, Corte Madera, CA). For statistical analysis of soma sizes, a two-tailed nonparametric Mann-Whitney $U$ test was used to evaluate the differences between population means. To determine the underlying distributions of cell soma size, we pooled the soma area data from the A laminae. We assumed for this analysis that the underlying distributions of neuronal soma sizes were distributed normally. We fit the histograms derived from these pooled data with gaussian functions, using the PStat program (Axon Instruments, Foster City, CA). Using a Simplex fitting algorithm, we achieved the best fit (least mean error per data point) for the Nissl data with two gaussian terms and with one gaussian term for the SMI-32 data. We evaluated with a comparison of $Z$ scores whether significant differences existed between the gaussian distributions derived from these fits.

\section{RESULTS}

\section{Nature of SMI-32 label in the normal LGN}

A number of observations suggest that SMI-32 staining in the normal LGN is restricted to the Y-cell population. First, the distribution of staining is similar to that of Y-cells (Sherman, 1985). As shown in Figure 1, cells are distributed in laminae A, $\mathrm{A} 1$, the magnocellular portion of $\mathrm{C}$, and the medial intralaminar nucleus (MIN), but the cells are lacking in parvocellular C laminae (C1-C3). Within the A laminae, the majority of the SMI-32-stained cells is near the laminar boundaries.

In addition, the morphology of SMI-32-stained cells is similar to that of Y-cells. As shown in Figures $1 C$ and 2, well stained cells exhibit class I morphology (Guillery, 1966; Friedlander et al., 1981; Wilson et al., 1984; Raczkowski and Sherman, 1985); they have large somata, with numerous radiating dendrites. SMI-32stained dendrites frequently cross the interlaminar zone between the A laminae as well as the boundary between the A1 and $\mathrm{C}$ laminae (Figs. $1 C, 2 C$ ).

Like Y-cells, SMI-32-stained cells are the largest in the LGN, as demonstrated by a comparison of the soma areas of cells stained for Nissl substance to those stained with the SMI-32 antibody (Fig. 3). The size of SMI-32-stained cells is significantly different from the overall population of LGN cells (Fig. $3 A ; n=$ 400 Nissl and 400 SMI-32; $p<0.0001)$ as well as cells in either laminae A or A1 (Fig. 3B, $C ; n=100$ Nissl and 100 SMI-32; $p<$ 0.0001 for lamina $\mathrm{A}$ and $\mathrm{A} 1$ ). In the magnocellular $\mathrm{C}$ lamina there is a slight, but significant, difference in the size distribution of SMI-32-stained cells and Nissl-stained cells (Fig. $3 D ; n=100$ Nissl and 100 SMI-32; $p<0.003)$. In the interlaminar zone between lamina A and A1 there was no significant difference between the sizes of SMI-32-stained and Nissl-stained cells (Fig. $3 E ; n=100$ Nissl and 100 SMI-32).

In addition to these comparisons, we also examined whether there were significant differences between the population of larger (presumably Y) cells and the SMI-32-stained neurons. The LGN A laminae contain three major types of cells: X- and Y-cells and interneurons. The soma size distributions of these populations overlap, which prompted our effort to fit gaussians to the data set in an attempt to derive the underlying distribution functions of these cells. However, we note that the best fits of the pooled A laminae Nissl data were achieved with two gaussian terms. This likely reflects that two of these types could not be discriminated with the sample size we used. Of the three, previous studies have shown the most overlap between X-cells and interneurons (Friedlander et al., 1981). Thus, the distribution of large Nissl-stained cells likely represents the distribution of Y-cells. As is apparent from the graphical comparison of gaussian distributions in Figure 4, the distribution of SMI-32-stained neurons was significantly different from that of the small Nissl-stained cells $(p<0.001)$, but not significantly different from the distribution of large Nissl-stained cells. Thus, the most reasonable interpretation of these data is that the antibody is labeling Y-cells.

Electron microscopic examination of SMI-32-stained tissue (lamina A) confirmed that label is found in somata and dendrites. Within somata the label displayed a patchy distribution that was not clearly associated with any particular organelle (Fig. $5 A$ ). The label was seen to coalesce as dendrites emerged from somata, and most dendritic staining was distributed evenly throughout the cytoplasm. In some cases, reaction product was denser toward synaptic contact zones or puncta adherentia (Fig. $5 B-E$ ).

A sample of 52 synaptic contacts made by retinal terminals onto SMI-32-stained dendrites was examined. Retinal terminals can be identified on the basis of their morphology as large terminals with round vesicles and pale mitochondria (RLP profiles). Previous studies indicate that Y-cells receive retinal contacts on proximal dendritic shafts; the synaptic arrangements of RLP profiles on Y-cells are generally less complex than those made by RLP profiles that innervate the dendritic appendages of X-cells (Wilson et al., 1984; Hamos et al., 1986, 1987; Bickford et al., 1992). 


\section{Soma Areas: LGN}

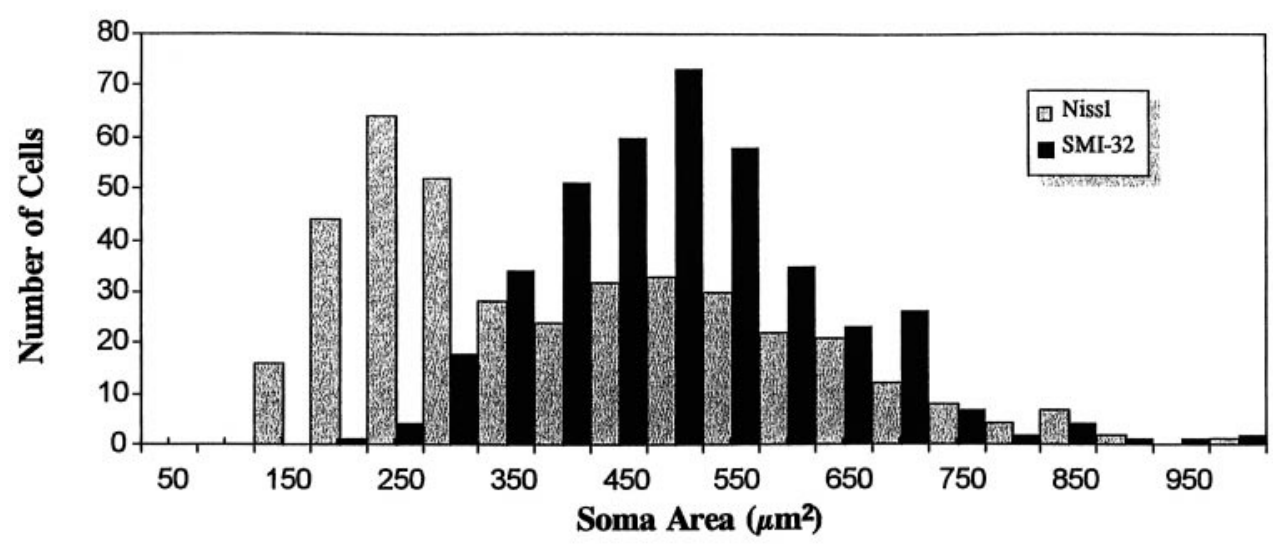

B

Layer A

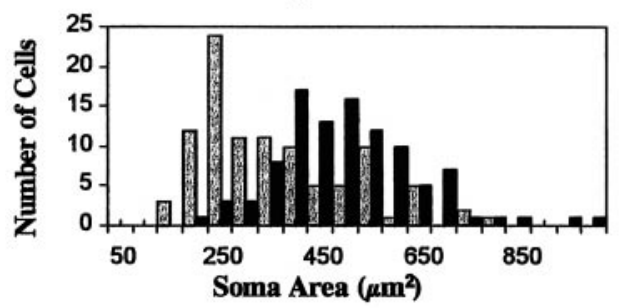
largest in the LGN. Histograms compare the distribution of soma areas of samples of Nissl- and SMI-32-stained cells in adjacent sections. $A$, Pooled data (400 Nissl- and 400 SMI-32stained cells) from all laminae. $B$, The 100 Nissl- and 100 SMI-32-stained cells from lamina A. $C$, The 100 Nissl- and 100 SMI-32-stained cells from lamina A1. D, The 100 Nissl- and 100 SMI-32stained cells from lamina C. $E$, The 100 Nissl- and 100 SMI-32-stained cells from the interlaminar zone between lamina A and A1.
D

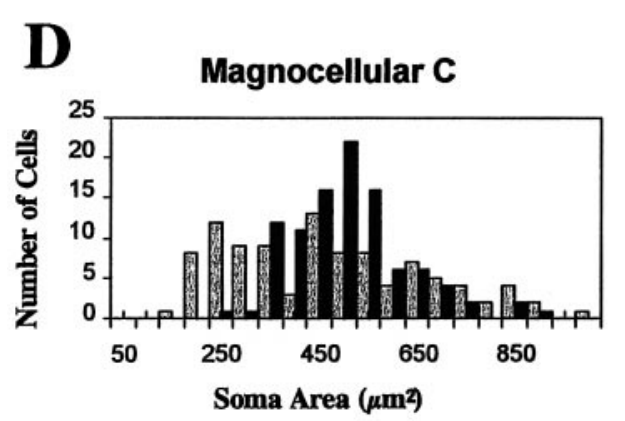

Consistent with the hypothesis that SMI-32 stains Y-cells, a majority of contacts between RLP profiles and SMI-32-stained dendrites were simple axodendritic contacts on dendritic shafts. Occasionally (13 of 52 or $25 \%$ ), retinal terminals presynaptic to SMI-32-labeled dendrites also contacted dendritic terminals (F2 profiles; Fig. $5 B$ ). However, only one triadic arrangement (frequently seen in the X-cell pathway; Wilson et al., 1984; Hamos et al., 1987) was encountered in the sample that was examined.

Axonal labeling with SMI-32 was also consistent with staining in Y-cells. Intensely stained axonal profiles were of large caliber and heavily myelinated (Friedlander et al., 1981; Humphrey et al., 1985a,b). These axons did not contain pale mitochondria and thus were not retinal axons. The distribution and size of these axons suggested that they were thalamocortical Y-cell axons, most likely originating from the SMI-32-stained cells. However, if this is the case, the staining did not extend far from the somata, because axonal labeling was not seen in the optic radiations.

\section{SMI-32 labeling after early monocular lid suture}

Because the SMI-32 antibody appears to stain Y-cells preferentially, it was of interest to examine staining with this antibody in cats raised with MS, a manipulation that affects the form and function of the Y-cell pathway. As illustrated in Figures 6 and 7, SMI-32 staining is reduced dramatically in geniculate A laminae deprived of normal visual input. The number of well stained cells and the general neuropil staining are both decreased in the deprived laminae when compared with the nondeprived laminae. Within the nondeprived laminae, cells with class I morphology continue to stain with the SMI-32 antibody. Within the deprived laminae, occasional cells that display class I morphology are labeled, but the staining intensity is decreased when compared with cells stained in the nondeprived lamina.

In contrast to the A laminae, the SMI-32 staining in lamina C is intense on both sides of the LGN of monocularly deprived cats. As previously described (Wiesel and Hubel, 1963; Guillery and Stelzner, 1970; Hickey, 1980; Murakami and Wilson, 1983, 1987), the deprived lamina $\mathrm{C}$ is slightly thinner than the nondeprived lamina C (Figs. 6-8). Nonetheless, both deprived and nondeprived lamina $\mathrm{C}$ cells are well stained with the SMI-32 antibody and display normal class I morphology. However, some differences were detected when the soma sizes of lamina $\mathrm{C}$ cells on 


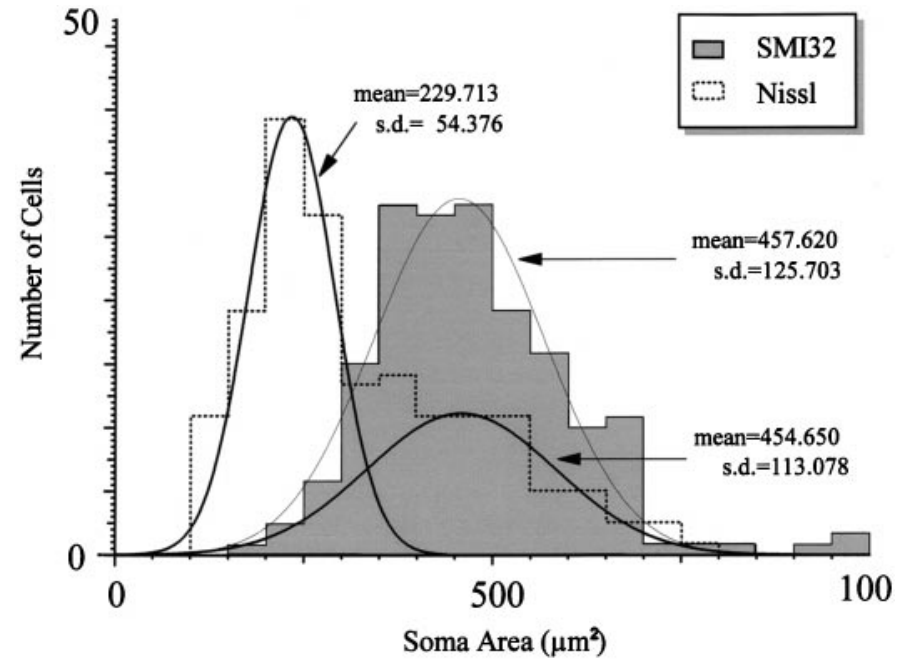

Figure 4. The soma areas of SMI-32-labeled cells are consistent with the population of Y-like cells. Histograms show the pooled data from the A laminae of LGN (see Fig. 3B,C). Gaussian functions were fit to the data by a Simplex least-squares algorithm. The two gaussians derived from the Nissl data significantly differ from each other, but the gaussians fitting the SMI-32 data and the larger population of Nissl-labeled cells were not significantly different.

either side of the LGN were compared. In each of the three cases the SMI-32-stained cells in deprived lamina $\mathrm{C}$ were slightly smaller (15\%) than those in the nondeprived lamina C. The size differences of samples of 100 SMI-32-stained cells in the deprived and nondeprived $\mathrm{C}$ laminae were significant in each of the three cases (Fig. 9; case 97-11, $p<0.001$; case 97-16, $p<0.0001$; case 97-17, $p<0.0001)$.

It is also apparent that, unlike other changes associated with MS, the reduction in SMI-32 staining occurs in both the binocular and monocular segments of lamina A. As previously reported for Nissl-stained sections (Guillery and Stelzner, 1970), cells in the deprived binocular segment are noticeably smaller than those in the monocular segment (Fig. 10A,B). However, in adjacent sections it is apparent that, although monocular segment cells have normal soma sizes, they do not retain their normal capacity to stain with the SMI-32 antibody (Fig. 10C,D). In contrast, the deprived monocular segment of lamina $C$ is stained with the SMI-32 antibody, indicated by a band of label beneath the monocular segment of lamina A.

\section{Alkaline phosphate pretreatment}

To begin to examine possible mechanisms that could account for the decrease in SMI-32 staining after monocular deprivation, we pretreated sections with alkaline phosphatase before immunocytochemical staining. This treatment has been used previously to reveal abnormal phosphorylation of neurofilaments (Su et al., 1996). As shown in Figure 11 $A$, SMI-32 staining in normal tissue revealed few axons because axonal neurofilaments normally are phosphorylated, and the SMI-32 antibody recognizes only nonphosphorylated neurofilament proteins. As shown in Figure $11 B$, dephosphorylation of the tissue before SMI-32 staining resulted in a dramatic increase in axonal staining. However, the alkaline phosphatase pretreatment did not affect the overall pattern of cellular staining in the monocularly deprived LGN. The nondeprived laminae served as an internal control for any effects of the alkaline phosphatase treatment on the intensity of cellular SMI-32 staining. After alkaline phosphatase pretreatment the
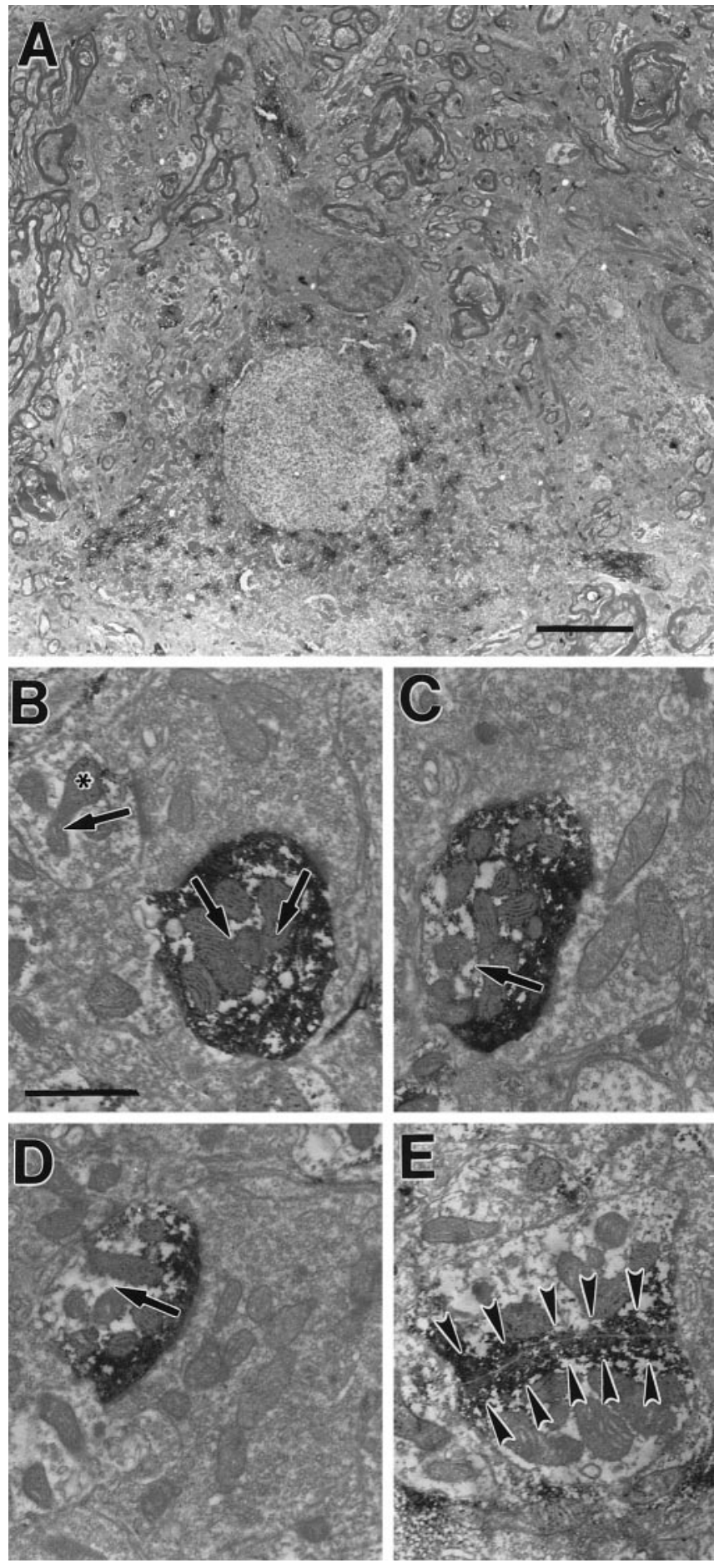

Figure 5. Ultrastructure of SMI-32 staining in the normal LGN. $A$, SMI-32 staining in cell somata is patchy and becomes more intense in dendrites. $B-D$, Simple axodendritic contacts (arrows) are made between retinal terminals and SMI-32-stained dendrites, although retinal terminals also may contact unlabeled dendritic terminals (asterisk in B). E, SMI-32 staining also is seen at puncta adherentia (arrowheads). Scale bars: in $A, 5 \mu \mathrm{m}$; in $B$ (also applies to $C-E$ ), $1 \mu \mathrm{m}$.

SMI-32 antibody stained cells in the nondeprived laminae. However, staining was still reduced in the deprived A laminae as compared with the nondeprived A laminae. Thus, it is unlikely that abnormal phosphorylation of neurofilaments accounts for the 

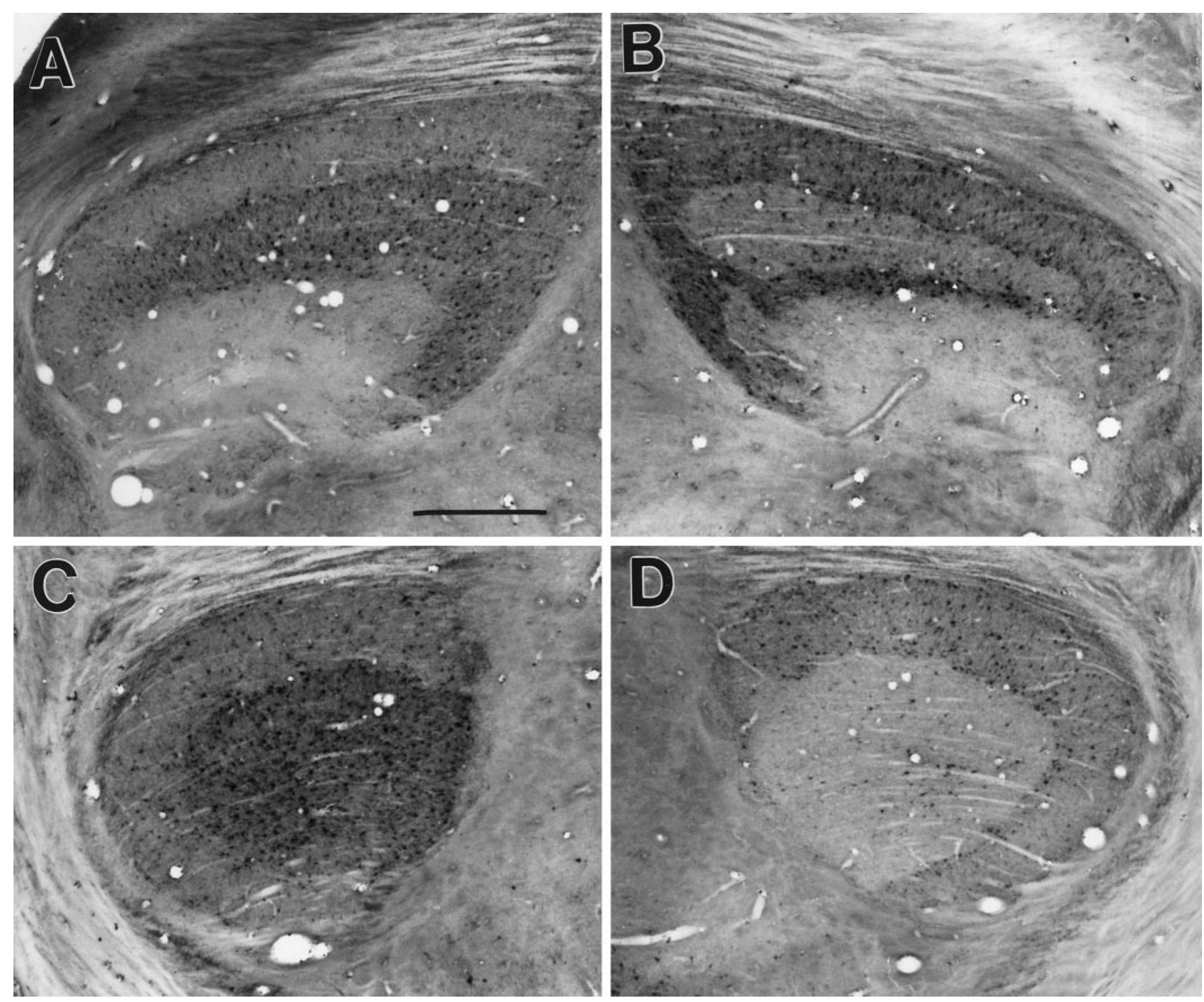

Figure 6. After MS, SMI-32 staining is reduced in the deprived A laminae. Shown are caudal $(A, B)$ and rostral $(C, D)$ sections through the LGN of a cat with right MS. In the left $\operatorname{LGN}(A, C)$, staining is reduced in lamina A. In the right LGN $(B, D)$, staining is reduced in lamina A1. Scale bar in $A$ (also applies to $B-D), 1 \mathrm{~mm}$.

loss of SMI-32 staining in LGN cells deprived of normal visual input.

\section{DISCUSSION}

\section{The SMI-32 antibody as a marker for Y-cells}

This study shows that the SMI-32 antibody preferentially stains Y-cells in the cat LGN. The distribution, morphology, and synaptic arrangements of LGN cells stained with this antibody match the characteristics of Y-cells. This is consistent with previous reports that the SMI-32 antibody preferentially stains the magnocellular layers in the monkey LGN (Gutierrez et al., 1995; Chaudhuri et al., 1996). In other areas of the brain, specific subsets of neurons stain with the SMI-32 antibody. In general, cells stained by this antibody are large, with thick dendrites and/or large-diameter axons. For example, the SMI-32 antibody stains the stout apical dendrites of cortical pyramidal cells (Campbell and Morrison, 1989; Carmichael and Price, 1994; del Rio and Defelipe, 1994; Cusick et al., 1995; Hof and Morrison, 1995; Hof et al., 1995). Within the visual system large cells in other structures are labeled. For example, the SMI-32 antibody labels a subset of large neurons in the pulvinar nucleus of the monkey (Gutierrez et al., 1995), and, in the retina, a relation to the Y-cell pathway has been demonstrated; in a variety of species SMI-32 or other neurofilament antibodies have been shown to stain large $\alpha$-ganglion cells (Drager et al., 1984; Gabriel and Straznicky, 1992; Straznicky et al., 1992).

\section{Evidence that neurofilament changes are attributable to cell class competition}

Two mechanisms have been proposed to account for the changes seen in the LGN after MS, namely, cell class and binocular competition. Cell class competition arises in laminae A and A1, where $\mathrm{X}$ and $\mathrm{Y}$ retinogeniculate arbors co-mingle and compete for terminal space on their geniculate cell counterparts. After MS, Y retinogeniculate arbors are reduced significantly, whereas the $\mathrm{X}$ retinogeniculate arbors maintain a broad terminal field (Sur et al., 1982; Garraghty et al., 1986). Thus, the Y-relay cells receive less, or abnormal, retinal input (Friedlander et al., 1982). Binoc- 

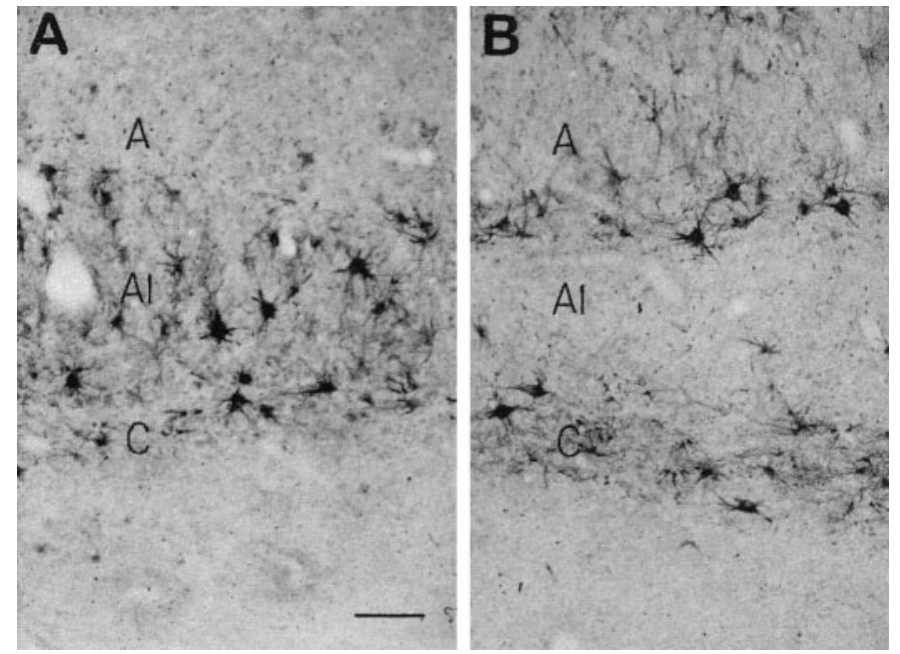

Figure 7. SMI-32-stained cells in C laminae and the nondeprived A laminae of MS cats display class I morphology. Shown is a section through the left $(A)$ and right $(B)$ LGN of a cat with right MS. Scale bar in $A$ (also applies to $B), 100 \mu \mathrm{m}$.
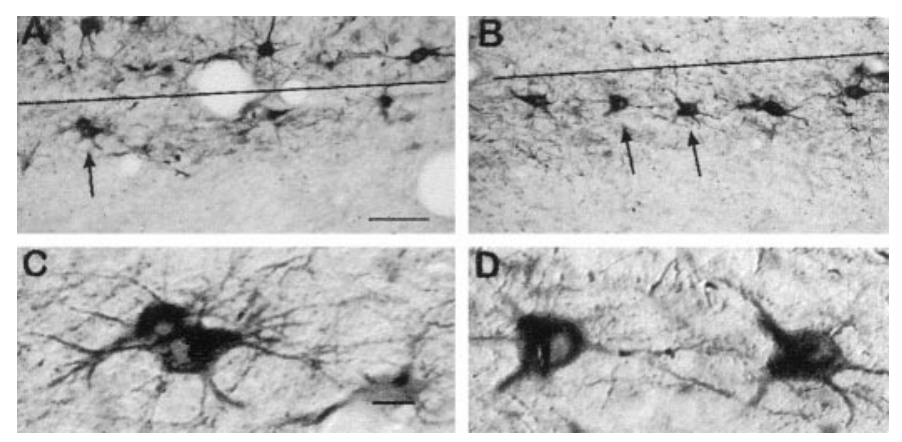

Figure 8. Cells in both the deprived and nondeprived $\mathrm{C}$ laminae stain with the SMI-32 antibody. Shown are the left $(A)$ and right $(B)$ C laminae of a cat with right MS. The borders between the A1 and C lamina are indicated by lines. The arrows point to cells shown at higher magnification in $C$ and $D$. Scale bars: in $A$ (also applies to $B$ ), $100 \mu \mathrm{m}$; in $C$ (also applies to $D), 20 \mu \mathrm{m}$.

ular competition arises in portions of the LGN where input from the two eyes innervate separate laminae. In this case, competitive mechanisms in the cortex result in reduced thalamocortical arbors arising from cells in the deprived laminae (Shatz and Stryker, 1978; Friedlander and Martin, 1991). This leads to a corresponding reduction in the soma sizes of cells in the binocular portions of the deprived laminae (Guillery and Stelzner, 1970; Guillery, 1972). It has been proposed that the abnormal properties of Y-cells seen in the binocular portions of the A laminae result from the combined effects of both cell class and binocular competition (Spear et al., 1989).

Our results suggest that the reduction in SMI-32 staining that accompanies MS is attributable to cell class competition and not to binocular competition. SMI-32 staining is reduced only in A-laminae, where $\mathrm{X}$ and $\mathrm{Y}$ retinogeniculate arbors compete for terminal space. In laminae $\mathrm{C}$, where cells are subject to binocular competition but not cell class competition, cells retain their ability to stain with the SMI-32 antibody. In the monocular zone of lamina A, where cells are subject to cell class competition but not binocular competition, SMI-32 staining is lost (Sherman and Spear, 1982; Spear et al., 1989).

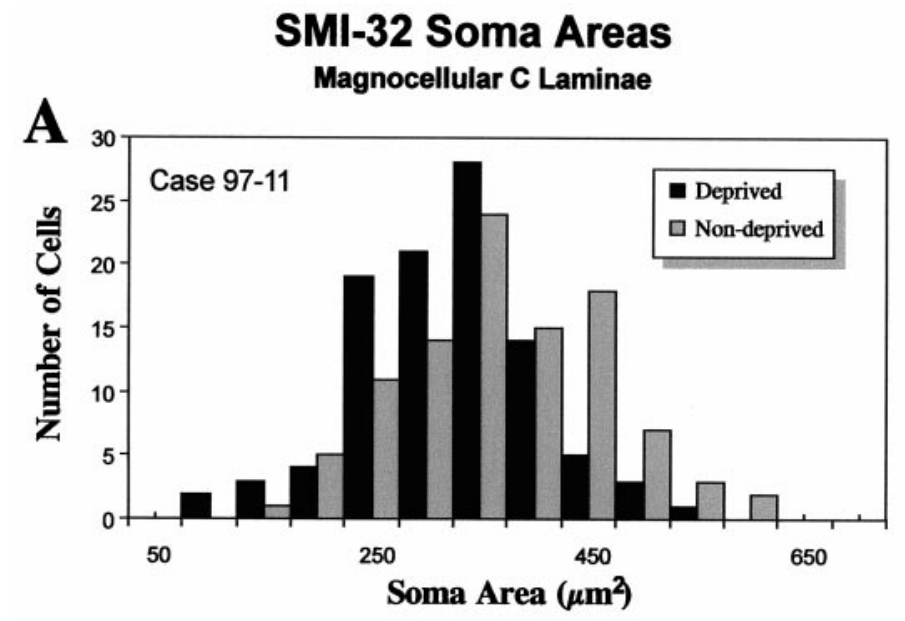

B
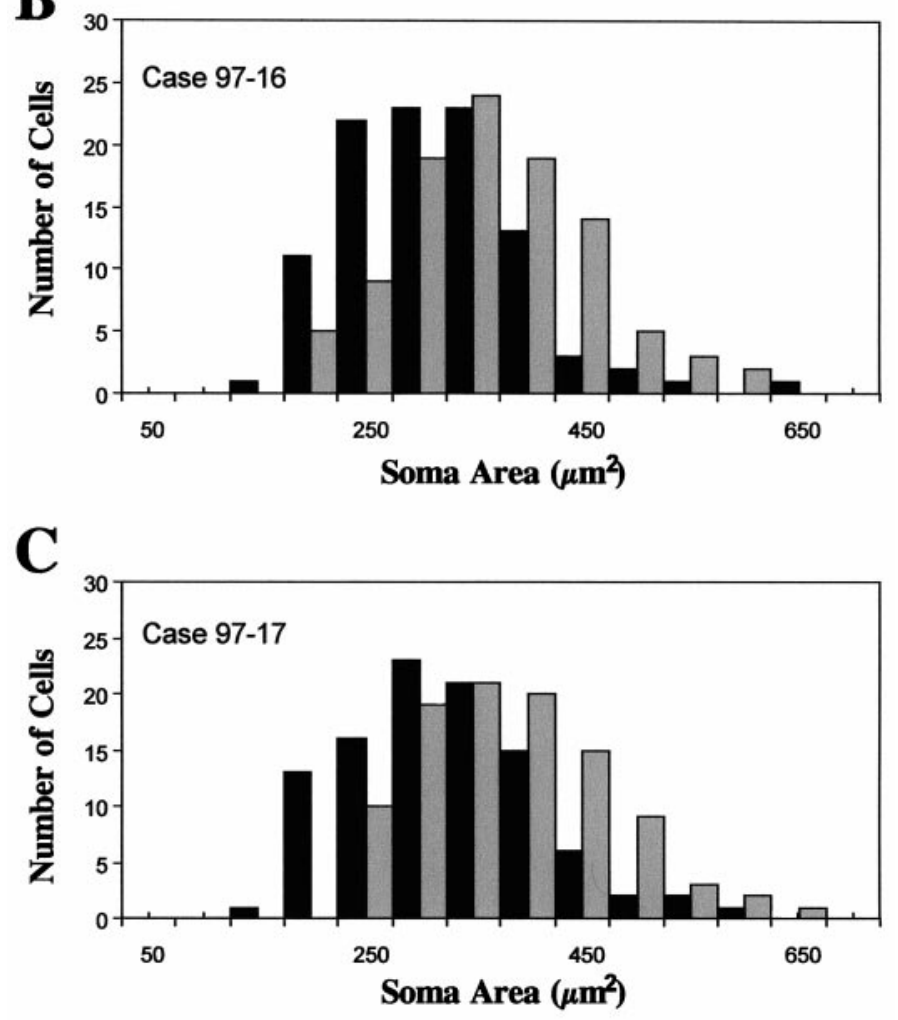

Figure 9. SMI-32-stained cells in the deprived lamina $\mathrm{C}$ are smaller than SMI-32-stained cells in the nondeprived lamina C. Histograms illustrate the soma size distributions of C lamina cells from three MS cats.

Indeed, these results also suggest that the consequences of MS on Y-cell morphology are attributable to different mechanisms. Changes in the cytoskeleton of Y-cells, which presumably account for the changes seen in dendritic morphology (Friedlander et al., 1982), appear to be attributable to cell class competition. In contrast, changes in soma size that appear to occur independent of the cytoskeletal changes are attributable mainly to binocular competition (Guillery and Stelzner, 1970; Guillery, 1972; Sherman et al., 1975). Thus, the nature of the input that Y-cells receive may have a major influence on the organization of their neurofilaments, whereas the extent of their axonal arbors influences their soma size. 

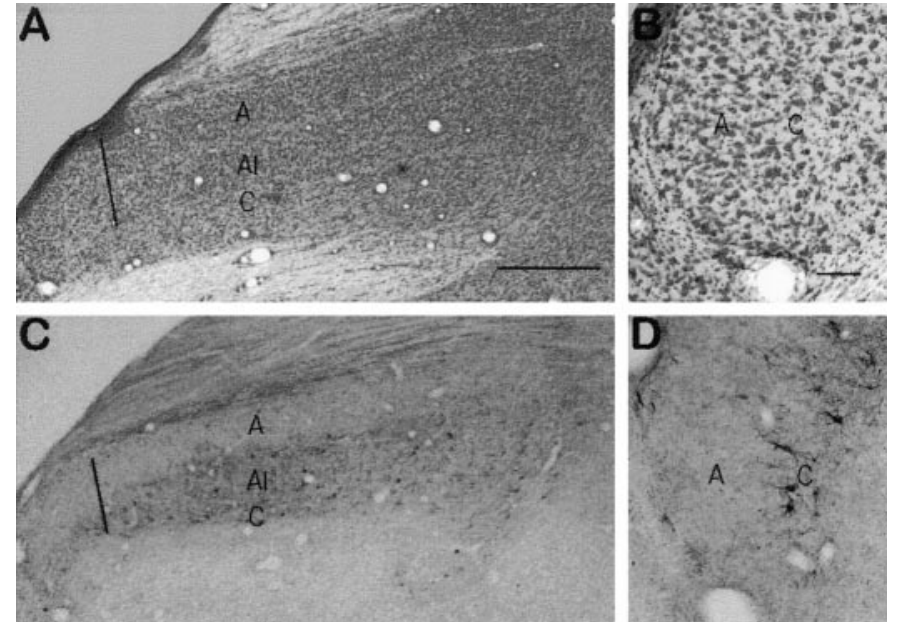

Figure 10. SMI-32 staining is reduced in both the binocular and monocular zones of the deprived A lamina. Shown are the Nissl-stained $(A, B)$ and SMI-32-stained $(C, D)$ sections through the left LGN of a cat with right MS. The border between the binocular and monocular zones is indicated by lines in $A$ and $C$. Higher magnifications of the deprived monocular zone are shown in $B$ and $D$. Monocular lamina $\mathrm{A}$ and $\mathrm{C}$ indicated. Scale bars: in $A$ (also applies to $C$ ), $500 \mu \mathrm{m}$; in $B$ (also applies to $D$ ), $100 \mu \mathrm{m}$.

\section{Modifications of the neuronal cytoskeleton}

Our results suggest that abnormal input to Y-cells induces a restructuring of their cytoskeleton. Other reported changes in neurofilaments, resulting from neurological disorders and after a variety of experimental conditions, indicate the dynamic nature of the neuronal cytoskeleton (Sternberger et al., 1985; Troncoso et al., 1986; Hof and Morrison, 1990; Hof et al., 1990; Vickers et al., 1992; Duong and Gallagher, 1994; Gai et al., 1994; Smith et al., 1995; Su et al., 1996). In the visual cortex of cats with monocular lid suture or monkeys with monocular tetrodotoxin injections, neurofilament staining becomes patchy (Eckert et al., 1997; Yoshioka, 1997), suggesting that cortical cells receiving input from the deprived eye also undergo cytoskeletal changes.

A well documented change in neurofilaments is abnormal phosphorylation. Generally, in adult tissue, neurofilaments in somata and dendrites are not phosphorylated, but those in axons are (Matus, 1988). The phosphorylation of neurofilaments in axons may allow the bundling of groups of neurofilaments, which confers compactness and stability to the axonal cytoskeleton. In Alzheimer's disease the neurofilaments in somata become abnormally phosphorylated, and this abnormal phosphorylation appears to accompany the formation of neurofibrillary tangles (Sternberger et al., 1985; Duong and Gallagher, 1994). Additionally, it is the cortical neurons that normally stain with the SMI-32 antibody that are particularly susceptible to cytoskeletal changes during Alzheimer's disease (Hof et al., 1990). One explanation for the vulnerability of SMI-32-positive neurons to cytoskeletal changes may be that the large $\mathrm{C}$ terminus of the NF-H protein has a high affinity for several kinases that phosphorylate the protein (Wible et al., 1989; Xiao and Montiero, 1994).

We ruled out abnormal phosphorylation of neurofilaments as a reason for the decreased SMI-32 staining of Y-cells in the deprived laminae, because removal of phosphate in sections pretreated with alkaline phosphatase did not alter the cellular staining pattern. A similar result was found in the substantia nigra of brains from patients with Parkinson's disease (Gai et al., 1994). Compared with the substantia nigra of control brains, the percent-
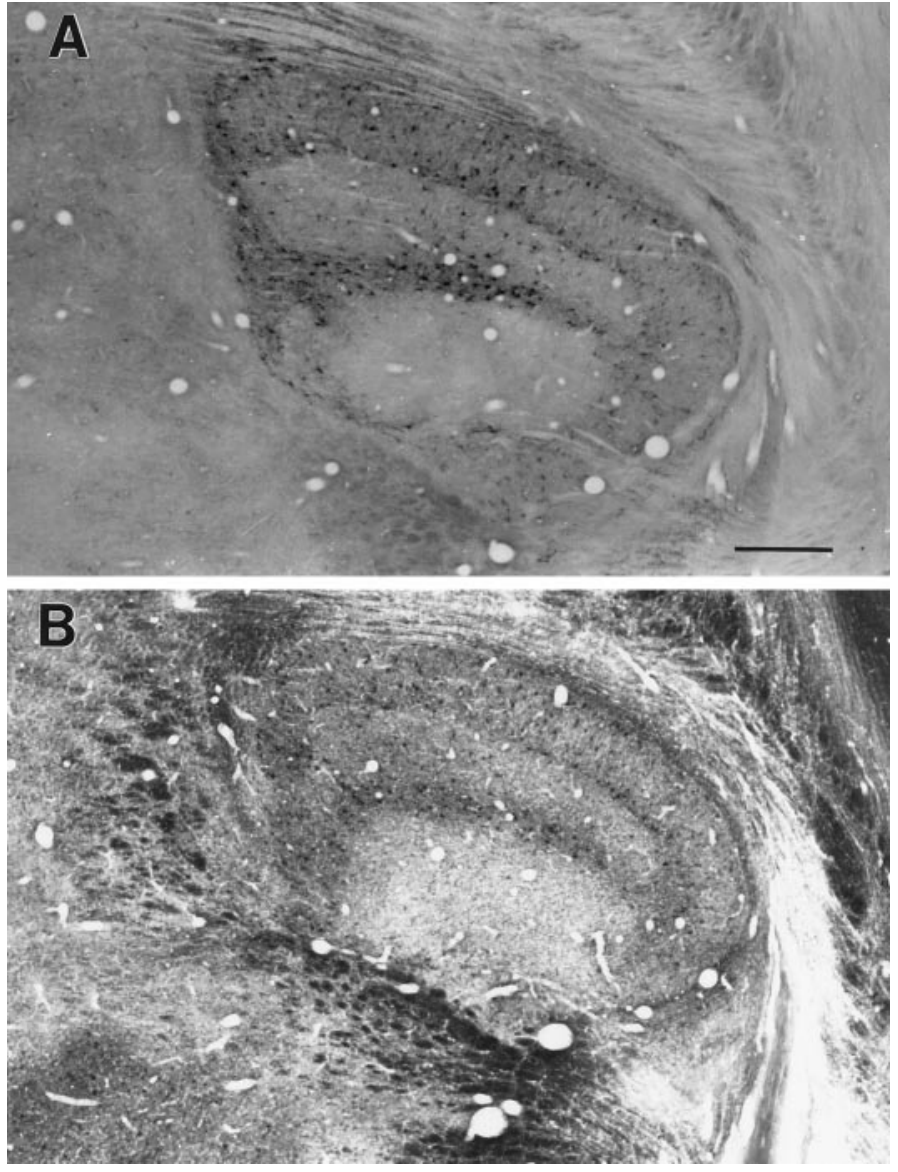

Figure 11. Alkaline phosphatase treatment before SMI-32 staining does not alter the cellular staining pattern in the LGN. SMI-32 staining is reduced in the right LGN A1 lamina of a cat with right MS $(A)$. Pretreatment of the tissue with alkaline phosphatase $(B)$ increases axonal labeling, but cellular staining remains reduced in the deprived lamina A1. Scale bar in $A$ (also applies to $B$ ), $1 \mathrm{~mm}$.

age of SMI-32-stained cells in the diseased brains was reduced, and this pattern remained in tissue pretreated with alkaline phosphatase. These authors suggested that the reduced SMI-32 staining might mark an initial stage of neuronal degeneration in which neurofilaments are one of the first proteins to be degraded.

Because LGN cells in deprived laminae do not degenerate, it is unlikely that the decreased SMI-32 staining shown in this study represents a process of neuronal degeneration. Thus, it remains to be determined whether deprived LGN Y-cells lack neurofilaments or whether their neurofilaments are reorganized such that they are not recognized by the SMI-32 antibody. In either case, our results demonstrate that changes in synaptic input can influence the organization of the neuronal cytoskeleton and suggest that the cat LGN offers a novel model system to study the normal function and pathological reorganization of neurofilaments.

\section{REFERENCES}

Adams JC (1981) Heavy metal intensification of DAB-based HRP reaction product. J Histochem Cytochem 29:775.

Bickford ME, Van Horn SC, Sherman SM (1992) Retinal Y axons contact interneurons in the lateral geniculate nucleus of the cat. Soc Neurosci Abstr 18:141.

Campbell MJ, Morrison JH (1989) Monoclonal antibody to neurofilament protein (SMI-32) labels a subpopulation of pyramidal neurons in the human and monkey cortex. J Comp Neurol 282:191-205.

Carmichael ST, Price JL (1994) Architectonic subdivision of the orbital 
and prefrontal cortex in the macaque monkey. $\mathrm{J}$ Comp Neurol 346:366-402.

Chaudhuri A, Zangenehpour S, Matsubara JA, Cynader MS (1996) Differential expression of neurofilament protein in the visual system of the vervet monkey. Brain Res 709:17-26.

Cusick CG, Seltzer B, Cola M, Griggs E (1995) Chemoarchitectonics and corticocortical terminations within the superior temporal sulcus of the rhesus monkey: evidence for subdivisions of superior temporal polysensory cortex. J Comp Neurol 360:513-535.

del Rio MR, Defelipe J (1994) A study of SMI-32-stained pyramidal cells, parvalbumin-immunoreactive chandelier cells, and presumptive thalamocortical axons in the human temporal neocortex. J Comp Neurol 342:389-408.

Drager UC, Edwards DL, Barnstable CJ (1984) Antibodies against filamentous components in discrete cell types of the mouse retina. J Neurosci 4:2025-2042.

Duong T, Gallagher KA (1994) Immunoreactivity patterns in neurofibrillary tangles of the inferior temporal cortex in Alzheimer's disease. Mol Chem Neuropathol 22:105-122.

Eckert MJ, Pegado VD, Duffy KR, Murphy KM (1997) Development of pyramidal neurons in cat visual cortex following normal or monocular visual experience. Soc Neurosci Abstr 23:2260.

Friedlander MJ, Martin KA (1991) Effects of monocular visual deprivation on geniculocortical innervation of area 18 in cat. $\mathbf{J}$ Neurosci 11:3268-3288.

Friedlander MJ, Lin C-S, Stanford LR, Sherman SM (1981) Morphology of functionally identified neurons in lateral geniculate nucleus of the cat. J Neurophysiol 46:80-129.

Friedlander MJ, Stanford LR, Sherman SM (1982) Effects of monocular deprivation on the structure-function relationship of individual neurons in the cat's lateral geniculate nucleus. J Neurosci 2:321-330.

Gabriel R, Straznicky C (1992) Immunocytochemical localization of parvalbumin and neurofilament triplet protein immunoreactivity in the cat retina: colocalization in a subpopulation of AII amacrine cells. Brain Res 595:133-136.

Gai WP, Vickers JC, Blumbergs PC, Blessing WW (1994) Loss of nonphosphorylated neurofilament immunoreactivity, with preservation of tyrosine hydroxylase, in surviving substantia nigra neurons in Parkinson's disease. J Neurol Neurosurg Psychiatry 57:1039-1046.

Garraghty PE, Sur M, Sherman SM (1986) The role of competitive mechanisms in the postnatal development of $\mathrm{X}$ and $\mathrm{Y}$ retinogeniculate axons. J Comp Neurol 251:198-215.

Garraghty PE, Shatz CJ, Stretavan DW, Sur M (1988) Axon arbors of X and Y retinal ganglion cells are differentially affected by prenatal disruption of binocular inputs. Proc National Acad Sci USA 85:7361-7365.

Guillery RW (1966) A study of Golgi preparations from the dorsal lateral geniculate nucleus of the adult cat. J Comp Neurol 128:21-50.

Guillery RW (1972) Binocular competition in the control of geniculate cell growth. J Comp Neurol 144:117-130.

Guillery RW, Stelzner DJ (1970) The differential effects of unilateral lid closure upon the monocular and binocular segments of the dorsal lateral geniculate nucleus in the cat. J Comp Neurol 139:413-422.

Gutierrez C, Yuan A, Cusick CG (1995) Neurochemical subdivisions of the inferior pulvinar in macaque monkeys. J Comp Neurol 363:545-562.

Hamos JE, Van Horn SC, Sherman SM (1986) Synaptic circuitry of an individual retinogeniculate axon from a retinal Y-cell. Soc Neurosci Abstr 12:1037.

Hamos JE, Van Horn SC, Raczkowski D, Sherman SM (1987) Synaptic circuits involving an individual retinogeniculate axon in the cat. J Comp Neurol 259:165-192.

Hickey TL (1980) Development of the dorsal lateral geniculate nucleus in normal and visually deprived cats. J Comp Neurol 189:467-481.

Hof PR, Morrison JH (1990) Quantitative analysis of a vulnerable subset of pyramidal neurons in Alzheimer's disease. II. Primary and secondary visual cortex. J Comp Neurol 301:55-64.

Hof PR, Morrison JH (1995) Neurofilament protein defines regional patterns of cortical organization in the macaque monkey visual system: a quantitative immunohistochemical analysis. J Comp Neurol 352:161-186.

Hof PR, Cox K, Morrison JH (1990) Quantitative analysis of a vulnerable subset of pyramidal neurons in Alzheimer's disease. I. Superior frontal and inferior temporal cortex. J Comp Neurol 301:44-54.

Hof PR, Nimchinsky EA, Morrison JH (1995) Neurochemical phenotype of corticocortical connections in the macaque monkey: quantitative analysis of a subset of neurofilament protein-immunoreactive pro- jection neurons in frontal, parietal, temporal, and cingulate cortices. J Comp Neurol 362:109-133.

Humphrey AL, Sur M, Uhlrich DJ, Sherman SM (1985a) Projection patterns of individual $\mathrm{X}$ - and $\mathrm{Y}$-cell axons from the lateral geniculate nucleus to cortical area 17 in the cat. J Comp Neurol 233:159-189.

Humphrey AL, Sur M, Uhlrich DJ, Sherman SM (1985b) Termination patterns of individual $\mathrm{X}$ - and $\mathrm{Y}$-cell axons in the visual cortex of the cat: projections to area 18 , to the $17 / 18$ border region, and to both areas 17 and 18. J Comp Neurol 233:190-212.

Matus A (1988) Neurofilament protein phosphorylation-where, when, and why. Trends Neurosci 11:7:291-292.

Murakami DM, Wilson PD (1983) Effects of early monocular deprivation on cells in the C-laminae of the cat lateral geniculate nucleus. Dev Brain Res 9:353-358.

Murakami DM, Wilson PD (1987) The development of soma size changes on cells in the C-laminae of the cat lateral geniculate nucleus following monocular deprivation. Dev Brain Res 35:215-224.

Raczkowski D, Sherman MS (1985) Morphology and physiology of single neurons in the medial interlaminar nucleus of the cat's lateral geniculate nucleus. J Neurosci 5:2702-2718.

Shatz CJ, Stryker MP (1978) Ocular dominance in layer IV of the cat's visual cortex and the effects of monocular deprivation. J Physiol (Lond) 281:267-283.

Sherman SM (1985) Functional organization of the W-, X-, and Y-cell pathways in the cat: a review and hypothesis. In: Progress in psychobiology and physiological psychology, Vol II (Sprague JM, Epstein AN, eds), pp 233-314. New York: Academic.

Sherman SM, Spear PD (1982) Organization of visual pathways in normal and visually deprived cats. Physiol Rev 62:738-855.

Sherman SM, Wilson JR, Guillery RW (1975) Evidence that binocular competition affects the postnatal development of Y-cells in the cat's lateral geniculate nucleus. Brain Res 100:441-444.

Smith MC, Mallory M, Hansen LA, Ge N, Masliah E (1995) Fragmentation of the neuronal cytoskeleton in the Lewy body variant of Alzheimer's disease. NeuroReport 6:673-676.

Spear PD, McCall MA, Tumosa N (1989) W- and Y-cells in the C layers of the cat's lateral geniculate nucleus: normal properties and effects of monocular deprivation. J Neurophysiol 61:58-73.

Sternberger NH, Sternberger LA, Ulrich J (1985) Aberrant neurofilament phosphorylation in Alzheimer disease. Proc National Acad Sci USA 82:4274-4276.

Straznicky C, Vickers JC, Gabriel R, Costa M (1992) A neurofilament protein antibody selectively labels a large ganglion cell type in the human retina. Brain Res 582:123-128.

Su JH, Cummings BJ, Cotman CW (1996) Plaque biogenesis in brain aging and Alzheimer's disease. I. Progressive changes in phosphorylation states of paired helical filaments and neurofilaments. Brain Res 739:79-87.

Sur M, Humphrey AL, Sherman SM (1982) Monocular deprivation affects $\mathrm{X}$ - and $\mathrm{Y}$-cell retinogeniculate terminations in cats. Nature 300:183-185.

Sur M, Weller RE, Sherman SM (1984) Development of X- and Y-cell retinogeniculate terminations in kittens. Nature 310:246-249.

Troncoso JC, Sternberger LA, Sternberger NH, Hoffman PN, Price DL (1986) Immunocytochemical studies of neurofilament antigens in the neurofibrillary pathology induced by aluminum. Brain Res 364:295-300.

Vickers JC, Delacourte A, Morrison JH (1992) Progressive transformation of the cytoskeleton associated with normal aging and Alzheimer's disease. Brain Res 594:273-278.

Wible BA, Smith KE, Anglelides KJ (1989) Resolution and purification of a neurofilament-specific kinase. Proc National Acad Sci USA 568:209-218.

Wiesel TN, Hubel DH (1963) Effects of visual deprivation on morphology and physiology of cells in the cat's lateral geniculate body. J Neurophysiol 26:978-993.

Wilson JR, Friedlander MJ, Sherman SM (1984) Fine structural morphology of identified X- and Y-cells in the cat's lateral geniculate nucleus. Proc R Soc Lond [Biol] 221:411-436.

Xiao J, Montiero MJ (1994) Identification and characterization of a novel (115 kDa) neurofilament-associated kinase. J Neurosci $14: 1820-1833$.

Yoshioka T (1997) Differential regulation of neurofilament triplet proteins under monocular deprivation in adult macaque V1. Soc Neurosci Abstr 23:1664. 